\title{
Factores para el estudio de la relación entre el grado de control familiar y el desempeño organizacional de las MIPyMEs familiares en el Área Metropolitana de Monterrey (Factors for the study of the relationship between the degree of family control and organizational performance of family MSMEs in Monterrey Metropolitan Area)
}

\author{
Armando Peña Hinojosa \& Joel Mendoza Gómez \\ Universidad Autónoma de Nuevo León, Facultad de Contaduría Pública y Administración, \\ San Nicolás de los Garza, N.L., México. \\ Email: armando_pena@hotmail.com
}

Keywords: family control, family-owned company, organizational performance

\begin{abstract}
The motors that move and drive the economies and societies of any country are the strength of the companies and might of the owners. At present, the importance of familyowned micro, small and medium sized companies is well known within the economies of the different Latin American and Caribbean countries; although, it is impossible to quantify exactly, the MSMEs are a key component in the contribution of economic growth, competitiveness, innovation, and in the generation of employment in any country; consequently, many governments and private institutions have initiated programs to promote this enterprising segment. It is not any different here in Mexico, as the above mentioned companies provide $50 \%$ of total employment and, in addition, $90 \%$ are family owned. This is where family influence or control becomes one of the important factors that have been proposed by the scientific academia as determining the organizational performance of a company. Based on this premise, the main topic of this article is to examine how the degree of family control affects the performance of said companies.
\end{abstract}

Palabras clave: control familiar, desempeño organizacional, empresa familiar

Resumen: Los motores que mueven e impulsan la economía, y por ende a las sociedades de cualquier país, son la fortaleza de las empresas y la pujanza de sus propietarios. En la actualidad, se conoce la importancia de las Micros, Pequeñas y Medianas Empresas (MIPyMEs) familiares dentro de las economías de los diferentes países de América Latina y el Caribe, a pesar de que no es posible determinarla en su exacta magnitud. Las MIPyMEs 
son una pieza clave en la contribución al crecimiento económico, a la competitividad, a la innovación y a la generación de empleos de los países; como consecuencia, muchos gobiernos e instituciones privadas han puesto en marcha políticas y programas para promover a este segmento empresarial. En México, esto no es diferente ya que brindan el $50 \%$ de los puestos de trabajo del país y además se calcula que el $90 \%$ de las empresas son de carácter familiar. Debido a lo anterior es qie el control o influencia familiar, llegar a ser uno de los factores más sustentados de los que han sido propuestos por parte de la academia científica como factores que determinan el desempeño organizacional de una empresa; con base en lo anterior, el principal aspecto de este artículo es examinar como el grado de control familiar de las familias propietarias afecta el desempeño organizacional de sus empresas.

\section{Introducción}

En el presente artículo se examina el hecho de cómo el control familiar afecta el desempeño organizacional de las empresas familiares del sector de las empresas familiares Micros, Pequeñas y Medianas Empresas (MIPyMEs) del área metropolitana de Monterrey, Nuevo León, México.

En la actualidad, se conoce la importancia de las MIPyMEs familiares dentro de las economías de los diferentes países de América Latina y el Caribe, a pesar de que no es posible determinarla en su exacta magnitud. Las MIPyMEs son una pieza clave en la contribución al crecimiento económico, a la competitividad, a la innovación y a la generación de empleos de los países; como consecuencia, muchos gobiernos e instituciones privadas han puesto en marcha políticas y programas para promover a este segmento empresarial.

En México, el sector de las MIPyMEs es un elemento fundamental para el desarrollo del país; la Secretaría de Economía indica que dichas empresas son responsables de más del $86 \%$ de los empleos formales así como del $68 \%$ del producto interno bruto INEGI (2010). Así mismo, según el Observatorio Pyme (CIPI de la SE, 2003), en el sector de las MIPyMEs, más de un $50 \%$ de éstas son catalogadas como empresas familiares.

En México se calcula que el $90 \%$ de las empresas son de carácter familiar y que más del $50 \%$ de las MIPyMEs son de tipo familiar, Kajihara (2004). De lo anterior es que se muestra la estratificación de empresas por tamaño, se establece con base en el sector económico al cual pertenecen, el número de empleados y el rango de ventas anuales, ver Tabla 1. 
Tabla 1. Estratificación de las empresas en México 2009.

\begin{tabular}{ccccc}
\hline Tamaño & Sector & $\begin{array}{c}\text { Rango de número } \\
\text { de trabajadores }\end{array}$ & $\begin{array}{c}\text { Rango de monto de } \\
\text { ventas anuales }(\mathrm{mdp})\end{array}$ & $\begin{array}{c}\text { Tope máximo } \\
\text { combinado }\end{array}$ \\
\hline Micro & Todas & Hasta 10 & Hasta $\$ 4$ & 4.6 \\
Pequeña & Comercio & De 11 hasta 30 & De $\$ 4.01$ hasta $\$ 100$ & 93 \\
Pequeña & Industria y Servicios & De 11 hasta 50 & De $\$ 4.01$ hasta $\$ 100$ & 95 \\
Mediana & Comercio & De 31 hasta 100 & De $\$ 100.01$ hasta $\$ 250$ & 235 \\
Mediana & Servicios & De 51 hasta 100 & De $\$ 100.01$ hasta $\$ 250$ & 235 \\
Mediana & Industria & De 51 hasta 250 & De $\$ 100.01$ hasta $\$ 250$ & 250 \\
\hline
\end{tabular}

Fuente: Tomado del Diario Oficial de la Federación, 30 de junio de 2009 (Secretaría de Economía, 2009).

Adicional a lo anterior, los motores que mueven e impulsan la economía, y por ende a las sociedades de cualquier país, son la fortaleza de las empresas y la pujanza de sus propietarios (en muchos casos, una familia). Aquí es donde la familia como propietaria de una empresa juega un papel muy importante en la economía, ya que la empresa familiar tiene un papel central en todo tipo de ellas, tanto de países desarrollados, como es el caso de EE.UU. o los países europeos, como en zonas en poco desarrolladas, como los países de América Latina o asiáticos Gersick (1997). En México esto no es diferente, Kajihara (2004):

- Brindan el $50 \%$ de los puestos de trabajo del país.

- Además se calcula que el $90 \%$ de las empresas son de carácter familiar.

En la actualidad nadie pone en tela de juicio la importancia de las MIPyMEs familiares dentro de las economías de los diferentes países de América Latina y el Caribe a pesar de que no es posible determinarla en su exacta magnitud. Las MIPyMEs son una pieza clave en la contribución al crecimiento económico, a la competitividad, a la innovación y a la generación de empleos. Como consecuencia, muchos gobiernos e instituciones privadas han puesto en marcha políticas y programas para promover a este segmento empresarial.

El efecto de la familia o el grado de control familiar son aquellos atributos o características que la familia propietaria de una empresa lleva a ella y que en determinado momento pueden afectar su desempeño organizacional. Dicho desempeño lo podemos definir ampliamente en términos de eficiencia, en términos de utilización de recursos de forma eficiente así como el cumplimiento de metas organizacionales, Steers (1982). Las familias propietarias afectan el desempeño organizacional de la empresa 
por medio de varios aspectos como son las metas propias de la familia, sus relaciones (con proveedores, accionistas, etc.) y los recursos o activos familiares que posee. Para desarrollar una teoría acerca de cómo las metas u objetivos familiares, las relaciones y los recursos afectan el desempeño organizacional de una empresa, lo más conveniente es partir de la teoría de agencia y de la teoría basada en los recursos, Chrisman, Chua, \&Litz (2004); estas dos teorías sugieren ciertos factores familiares pueden acarrear beneficios de agencia e importantes activos, mientras otros factores familiares imponen costos que se convierten en pasivos y son barreras para el óptimo desempeño de la empresa

En contraste, pese a la gran importancia MIPyMEs familiares en México, es poca la investigación científica que se ha realizado sobre ellas. De acuerdo al análisis previo, la influencia de la familia o grado de control familiar se define como punto importante en el análisis del desempeño organizacional de una empresa; el analizar (en qué consiste dicho factor, como se caracteriza y que teorías lo soportan) y predecir cómo la afectan en la consecución o logro de sus metas y objetivos se vuelve una cuestión crítica y de alta prioridad para la administración o dirección general de la misma.

Con lo anterior expuesto, el problema de investigación identificado es el siguiente: se desconoce como el grado de control familiar afecta el desempeño organizacional en las MIPyMEs familiares en México.

En este contexto surge la siguiente pregunta de investigación: ¿Está el grado de control familiar relacionado con el desempeño organizacional de las empresas MIPYME familiares del área metropolitana de Monterrey?.

En consecuencia de lo anterior el objetivo general de la investigación es demostrar que el grado de control familiar es un factor crítico de éxito del desempeño organizacional de las empresas MIPYME familiares en el área metropolitana de Monterrey, examinándolo y analizándolo para ver su influencia en él.

\section{Antecedentes teóricos generales del tema}

En esta sección se profundizará en el análisis teórico de diversos autores acerca de los conceptos de la familia, la empresa familiar y la 
importancia de la empresa MIPyME, el desempeño organizacional y el grado de control familiar.

Antes de comenzar a profundizaren el concepto e importancia de la empresa familiar debemos de analizar primero que es la familia, como es la familia en México, como son sus principales características y que cambios ha tenido como consecuencia de la evolución de la sociedad mexicana.

\section{La familia en México}

La familia es el grupo o entidad social básica en el que la mayoría de la población se organiza para satisfacer sus necesidades esenciales; es el marco de referencia en donde los individuos nacen y se desarrollan, así como el contexto donde se construye la identidad de las personas por medio de la transmisión y actualización de los patrones de socialización. Según la Declaración Universal de los Derechos Humanos de 1948, la familia es el elemento natural y fundamental de la sociedad y tiene derecho a la protección de la sociedad y del Estado, INEGI (2010).Como forma elemental o primaria de agrupamiento u organización de la humanidad, la familia es el elemento básico de referencia para cualquier ser humano, Fabelo (2001).

Algunos autores extienden el concepto de familia al ámbito sistémico, definiéndola como un sistema integrador multigeneracional dinámico viviente, el cual está sometido a una continua dinámica de generación de reglas y de búsqueda de acuerdos entre ellas, González (2007). Adicionalmente indica que la familia puede ser vista como un agente cibernético, caracterizado por poseer una gran variedad de subsistemas (así define a los lazos o relaciones que se establecen entre esposos, entre padres e hijos, entre hermanos, etc.) de funcionamiento interno e influido por agentes externos relacionados a ella.

La familia es el grupo o entidad social básica en el que la mayoría de la población se organiza para satisfacer sus necesidades esenciales; es el marco de referencia en donde los individuos nacen y se desarrollan, así como el contexto donde se construye la identidad de las personas por medio de la transmisión y actualización de los patrones de socialización. Según la Declaración Universal de los Derechos Humanos en 1948, la familia es el elemento natural y fundamental de la sociedad y tiene derecho a la protección de la sociedad y del Estado, (INEGI, 2010). 
También se indica que la familia mexicana se caracteriza por una fuerte conservación de las tradiciones, las cuales son un reflejo de la importancia de la familia nuclear; otros aspectos a considerar son su apego a la colectividad y a las creencias religiosas, Rendón (2010). Se debe señalar que señalar también que los cambios en la estructura y comportamientos familiares son diversos al interior del país debido a la alta heterogeneidad que lo caracteriza. Con frecuencia las conductas que se presentan dentro del núcleo familiar son transmitidas al lugar del trabajo, Belausteguigoitia (2005).

La familia mexicana se caracteriza por su integración y su contante crecimiento como es el caso de la familia extendida, además de la integración de amigos y visitantes.

Algunas de sus principales características son como lo indican Morales (2009) y Rendón (2010):

- Conservación de tradiciones.

- Establecimiento de vínculos y lazos familiares muy fuertes.

- Que en el hogar extendido vivan más de una familia nuclear, formando así una familia extendida (hogares con hijos casados y sus familias).

- Cohesión y solidaridad entre sus miembros.

- De esto último se origina la influencia de la familia hacia la empresa familiar. Aquí es donde versa la importancia de la familia, dado que es la parte fundamental en la que se origina la empresa familiar.

\section{La empresa familiar}

En la literatura de investigación del tema, se han encontrados diferentes características que debe de tener una empresa para ser llamada empresa familiar. Como lo indica Gibb (2006), hay algunas investigaciones en las que se define el concepto de empresa familiar de una manera subjetiva, sin bases teóricas o científicas, mientras que otros investigadores basan sus clasificaciones en criterios más objetivos, tales como el porcentaje de la propiedad de una empresa, o el número de miembros de la familia que ocupan posiciones directivas en la empresa, o que ocupan posiciones en el consejo de administración. Esta diversidad de ideas lleva a tener clasificaciones diferentes, incluso en algunos casos contradictorias.

De acuerdo a lo presentado por Poza (2007), una empresa familiar se sintetiza en las siguientes características: 
- Hay un alto porcentaje del control de la empresa ( $15 \%$ o más) sostenido por uno o dos miembros de una familia, o de una asociación de familias.

- La empresa tiene la visión (o el deseo) declarada de continuar operando en el tiempo, a través de las generaciones futuras.

- La empresa se caracteriza por tener una alta preocupación por las relaciones de negocios de los miembros de la familia.

- Hay una gran influencia familiar estratégica en la administración de la empresa, ya sea por una posición activa en la gerencia o dirección de la empresa, o porque los miembros de la familia forman parte del consejo de administración, o son accionistas de la empresa.

En Estados Unidos de América, de acuerdo con McConaughy, Mendoza, \&Mishra (1996), las bases que se tomaron en cuenta para definir a las empresas familiares que integran el índice de Loyola University Chicago Family Firm Stock Index (LUCFFSI, por sus siglas en ingles de) son las siguientes:

- Que fueran controladas y/o administradas por el fundador o por algún miembro de la familia fundadora

- Que miembros de la familia fundadora se encontraran ocupando puestos en el equipo de directores, o en el consejo de administración de la empresa.

De acuerdo a lo presentado por Martínez, Stöhr, \& Quiroga (2007) en Chile, una empresa familiar está definida por uno o más de los siguientes criterios:

- Es una empresa cuya propiedad está controlada por una familia (más de la mitad de los miembros del consejo de administración son familiares entre sí). Adicionalmente, se tiene que los miembros de dicha familia participan como miembros de la plantilla de directores y/o en la alta administración de la empresa.

- Es una empresa cuya propiedad está controlada por un grupo de dos o más familias, cuyos integrantes forman parte de más de la mitad de los miembros del consejo de administración. Adicionalmente, se tiene que los integrantes de dichas familia participan como miembros de la plantilla de directores y/o en la alta administración de la empresa. 
También en Chile se clasificaron a las empresas familiares de acuerdo a los siguientes criterios, Bonilla, Sepúlveda, \& Carvajal (2010):

- Examinaron la lista de grupos corporativos de negocios o comerciales publicados por la Superintendencia de Valores y Seguros de Chile (SVS), sí un grupos de los ahí enlistados se podían asociar a una familia de tradición empresarial, las compañías que constituían ese grupo se clasificaban como empresas familiares.

- Si una compañía no era miembro de los grupos corporativos indicados en el punto anterior, se le podía clasificar como empresa familiar si su dirección o gerencia general estaba controlada por uno o más miembros de una familia listada en el SVS.

- Si una compañía no era miembro de los grupos corporativos indicados en primer punto, ni correspondía al segundo criterio, se le podía clasificar como empresa familiar si el equipo de directores estaba controlado por uno o más miembros de una familia listada en el SVS.

Lo anterior coincide parcialmente, y se complementa con lo indicado por Villalonga \& Amit (2004) y Allouche, Amann \& Kurash (2008) quienes comentan que para que una empresa sea considerada empresa familiar debe de incluir cuando menos tres de las características siguientes:

- Una o más familias mantienen una parte significativa del capital de la empresa.

- Los miembros de la familia mantienen un control significativo (al menos un $20 \%)$ sobre las decisiones de la compañía, de las cuales depende la distribución del capital y los derechos de votos de los miembros del consejo que no son miembros de dicha familia.

- Los miembros de la familia se desempeñan como altos directivos dentro de la organización de la empresa en cuestión.

Por otro lado, en Estados Unidos de América, Astrachan\& Carey (2003), define la empresa familiar con la siguiente clasificación de categorías. Categoría 1:

- Tiene múltiples generaciones de la misma familia en la historia de la empresa.

- Mas un miembro de la familia propietaria de la compañía, ocupa una posición en la administración de la misma. 
Categoría 2:

- El fundador o sus descendientes administra la compañía.

- Se intenta mantener el control de la compañía familiarmente.

Categoría 3:

- Gran participación de la familia.

- Una familia tienen el control estratégico de la compañía.

En las investigaciones realizadas en Italia, Prencipe, Markarian \& Pozza (2008) definen a la empresa familiar como:

- Es una compañía en la cual hay una o más familias enlazadas por un parentesco, una cercana afinidad, o una alianza sólida, tienen un gran monto de capital que los habilita para poder tomar decisiones estratégicas para definir el rumbo de dicha compañía.

- Se caracteriza por tener una cercana relación entre los directivos de la empresa y la familia que la controla.

Para el caso de México, Belausteguigoitia (2005) indica que se acepta como empresa familiar a aquella organización controlada (propiedad) por una familia, donde dos 0 más miembros de ella trabajan activamente en esa organización. En general, y coincidiendo con Grabinsky (1991), podemos concluir que una empresa familiar es aquella en la que sus dueños, así como quienes la dirigen u operan, forman parte de una o varias familias, queriendo decir lo anterior, que el control de la empresa encuentra en manos de esos miembros de la(s) familia(s). Un resumen de los diferentes definiciones de empresa familiar hechas por investigadores del tema a nivel mundial se presenta a continuación, ver Tabla 2.

Para la presente investigación definiremos que una empresa familiar tiene las siguientes características:

- Una o más familias mantienen una parte significativa del capital de la empresa.

- Varios de la(s) familia(s) mantienen un control significativo y/o se desempeñan como altos directivos dentro de la organización. 
Tabla 2. Resumen de conceptos de empresa familiar

\begin{tabular}{|c|c|c|c|}
\hline Autor & Año & País & Una Empresa es Familiar sí: \\
\hline McConaughy & 1996 & USA & $\begin{array}{l}\text { Son controladas y/o administradas por el fundador o por algún } \\
\text { miembro de la familia fundadora. }\end{array}$ \\
\hline Astrachan & 2003 & USA & $\begin{array}{l}\text { Tiene múltiples generaciones de la misma familia en la historia de } \\
\text { la administración de la empresa. }\end{array}$ \\
\hline Villalonga & 2004 & USA & $\begin{array}{l}\text { Una o más familias mantienen una parte significativa del capital } \\
\text { de la empresa. Miembros de la familia mantienen un control } \\
\text { significativo (al menos un } 20 \% \text { ) sobre las decisiones de la } \\
\text { compañía. }\end{array}$ \\
\hline Martínez & 2007 & Chile & $\begin{array}{l}\text { Está controlada por una familia (son familiares entre sí más de la } \\
\text { mitad de los miembros del consejo de administración). Sí se } \\
\text { observa que los miembros de dicha familia participan como } \\
\text { miembros de la plantilla de directores y/o en la alta administración } \\
\text { de la empresa. }\end{array}$ \\
\hline Allouche & 2008 & Francia & $\begin{array}{l}\text { Una o más familias mantienen una parte significativa del capital } \\
\text { de la empresa (al menos un } 20 \% \text { ). }\end{array}$ \\
\hline Belausteguigoitia & 2009 & México & $\begin{array}{l}\text { Está controlada por una familia, donde dos o más miembros de } \\
\text { ella trabajan activamente en ella. }\end{array}$ \\
\hline Bonilla & 2010 & Chile & $\begin{array}{l}\text { Sí un grupo de la lista de Valores y Seguros de Chile (SVS) se le } \\
\text { pude asociar a la empresa. Sí el equipo de directores estaba } \\
\text { controlado por uno o más miembros de una familia listada en el } \\
\text { SVS. }\end{array}$ \\
\hline
\end{tabular}

Fuente: autoría propia.

\section{Importancia de la empresa familiar.}

Desde el punto de vista económico, para el caso de Estados Unidos de América, Poza (2007) menciona que empresas familiares representan aproximadamente el $90 \%$ de todos los negocios corporativos, lo que se traduce en aproximadamente de 17 millones de empresas operando. Por otro lado, también menciona que en ese país, un tercio de las 500 compañías más poderosas indicadas por la revista Fortune en su edición del año 2005, son empresas familiares, además de que cerca de un $60 \%$ de las empresas que cotizan en la bolsa tienen una gran influencia de tipo familiar. Así mismo, indica que en Estados Unidos de América, las empresas familiares representan aproximadamente el $60 \%$ del PIB, $85 \%$ del empleo en el sector privado y cerca del $86 \%$ de todos los trabajos creados en la década pasada.

Por su parte Anderson (2003) en su estudio hecho también en Estados Unidos de América con las empresas listadas el reporte de Standard\&Poors 
(S\&P) 500 de los años 1992 a 1999, encontró que las empresas familiares tienen significativamente un mejor performance organizacional que las que no lo son; sus resultados mostraron que los beneficios obtenidos son mayores en empresas familiares (en comparación con las que no lo son) basándose en aquellas empresas en las cuales un miembro de la(s) familia(s) funge como CEO.

En Japón, Allouche, Amann \& Kurash (2008) resaltan la importancia tradicional de la empresa familiar. Este tipo de empresas comenzaron a operar mucho antes de la apertura de este país hacia el mundo, ocurrido a finales del siglo XIX. También en este mismo país Kurashina (2003) encontró que las empresas familiares tienen en promedio un mejor desempeño organizacional que las empresas no familiares.

Para el caso de Latinoamérica, Martínez, Stöhr, \& Quiroga (2007) decidieron hacer su investigación en Chile ya que ese país ofrece un esquema de proteccionismo gubernamental diferente al de Estados Unidos y al de los países asiáticos. Ellos encontraron que en Chile las empresas familiares tienen un mejor desempeño organizacional que las empresas no familiares, siendo, esto congruente con lo encontrado por Anderson (2003) Así mismo, también para el caso de Chile, Bonilla, Sepulveda, \& Carvajal (2010) encontraron que las empresas familiares no sólo obtienen mejores beneficios comparadas con las que no lo son, sí no que además son menos riesgosas.

Para el caso de México y América Latina Belausteguigoitia (2005), resalta la importancia económica de las MIPyMEs. Indica que en la mayoría de los estudios examinados por él, la proporción máxima de las empresas familiares respecto de las no familiares es de aproximadamente un $90 \%$, es decir 9 de cada 10 empresas son familiares. Además, indica las ventajas que tiene una empresa familiar:

- Las relaciones de afecto son la gran fortaleza de este tipo de empresas.

- El compromiso hacia la organización.

- La visión de largo plazo.

- La vocación y oficio de la familia dueña.

- La rapidez en la toma de decisiones.

- La rotación de directivos es menor. 
Respecto de las ventajas que tiene la empresa familiar, en la literatura se indica que puede tener dos fortalezas básicas (Gallo, 1995):

- Respecto de la unidad:

- Intereses comunes.

- Autoridad reconocida.

- Confianza mutua.

- Comunicación.

- Respecto del compromiso:

- Sacrificio personal

Exigencia de lo mejor

- Pensamiento a largo plazo

A continuación en esta sección del marco teórico revisaremos los aspectos teóricos del desempeño y del grado de control familiar así como los estudios empíricos que se han realizado para mostrar su relación.

\section{El desempeño organizacional}

En esta sección se definirá el concepto con el cual estaremos haciendo uso del desempeño organizacional revisando por una parte algunas teorías del desempeño organizacional y por otro parte algunos conceptos para definirlo.

\section{Algunas de teorías del desempeño organizacional}

Dentro de las teorías que tratan el desempeño organizacional de una empresa tenemos la teoría es la teoría de sistemas. Esta teoría está basada en el hecho de que hay muchos componentes de una organización 0 un grupo que constituyen un sistema. Establece que cada componente de un sistema es interdependiente, en un grado algunas veces mayor o menor, de otros componentes del sistema y que todos ellos tendrían algún tipo de impacto si el sistema cambia en algún aspecto, Wallace (2010).

La base de la teoría de sistemas define el hecho de que cada sistema incluye subsistemas, reglas, cambios, metas y la habilidad para llevar a cabo sus metas por medio de una variedad de métodos o formas. Basado en lo 
anterior, se asumirá que la familia (propietaria de una empresa) es, en sí misma, un sistema tal como lo es la empresa; esta teoría se usará para explorar como el grado de control familiar afecta el desempeño organizacional de una empresa familiar.

Adicionalmente también mencionan la teoría de contingencia, la cual indica que las empresas u organizaciones deben de tratar de manejar el nivel de incertidumbre del entorno por medio de la implementación estrategias, tanto internas y como externas. Entre las estrategias internas se encuentra la definición de proceso y métodos así como estructuras organizacionales que se adecuen en forma perfecta a las condiciones ambientales. Se busca el contar con estructuras orgánicas, flexibles y planas para aquellos entornos de alta complejidad y dinamismo, y estructuras mecanicistas y rígidas para aquellos entornos menos cambiantes e inciertos. De acuerdo a Pinto (2003) esta teoría establece que ni en las organizaciones ni en la teoría administrativa no hay nada absoluto, todo es relativo y siempre está en función de algún factor, ya sea interno o externo a dicha organización.

En este mismo camino, se ha buscado explicar cómo el entorno afecta el desarrollo de ventajas competitivas y el cumplimiento de los objetivos de las organizaciones (es decir, su desempeño organizacional); cómo las organizaciones tratan con el dinamismo, la complejidad, la incertidumbre y el grado de predictibilidad del medio ambiente o entorno organizacional. Los investigadores que han estado trabajando en clarificar como es la organización-entorno-desempeño lo han realizado trabajando en las siguientes teorías, Fuentes (2005): teoría de redes sociales, teoría basada en los recursos, teoría ecológica poblacional, teoría institucional, teoría de los costos económicos de transacción y teoría de juegos.

Teoría de redes sociales (social network theory). La Teoría de redes sociales es una rama de la Microeconomía que busca como poder predecir el resultado del comportamiento agregado de un grupo de personas definido por las relaciones entre sus miembros, en este caso el cómo un grupo de individuos laborando en una empresa pueden contribuir al buen desempeño organizacional de la misma. Los principios centrales en la teoría de redes sociales, independientemente de las diferencias que puedan darse entre distintos analistas, son los siguientes, Lozares (1996): 
- Los actores y sus acciones son contemplados como interdependientes y no como independientes o unidades autónomas.

- Los lazos relacionales entre los actores vehiculan transferencias de recursos, tanto materiales como no materiales.

- Los modelos contemplan las estructuras de relaciones como entornos que o bien proporcionan oportunidades o bien coaccionan la acción individual.

- Los modelos de redes identifican la estructura social, económica, política, etc. como pautas constantes de relaciones entre actores.

La teoría basada en los recursos (Resource-Basedview theory) tiene su base en las ideas básicas que fueron formuladas por Penrose (1959), Wernerfelt (1984) y Barney (1991), como una perspectiva desde el exterior en oposición al punto de vista basado desde el mercado (considera como una en perspectiva desde el interior). Esta teoría se basa en la asunción de que una ventaja competitiva es resultado de una óptima combinación y asignación de recursos en escenarios imperfectos (con muchas características adversas). Estos recursos pueden ser activos, habilidades, y capacidades (de organización), teniendo todos las características que todos ellos que llevan a las ventajas competitivas tienden a ser escasos, valioso, insustituibles y difíciles de ser imitables. Muchas derivados de esta teoría son ahora conocidas, como el caso de la teoría de las capacidades dinámicas (Dynamic Capabilities Theory) y la teoría de la firma basada en el conocimiento (knowledge-based theory of the firm). Todas estas teorías derivadas tienen el mismo acercamiento o aproximación básica con respecto a la formulación de una estrategia corporativa. La esencia de la formulación de la estrategia es entonces el diseñar una estrategia que haga el uso más eficiente de los recursos y capacidades de la base para poder obtener el mejor desempeño organizacional, Müller-Lietzkow (1991).

Respecto de la teoría de recursos y capacidades, ha sido aplicada para describir un modelo que explica la forma como el desempeño de una empresa está en función de las riquezas y valores agregados que puedan ser creados a partir de las sinergias que puedan surgir cuando los recursos y capacidades de los individuos, de la familia y la empresa, son enfocados a un mismo objetivo, Cibrián (2009).Un resumen de las teorías de desempeño organizacional está a continuación, ver Tabla 3. 
Tabla 3. Resumen de teorías de desempeño organizacional

\begin{tabular}{|c|c|c|}
\hline Teoría & Descripción & Autores \\
\hline Teoría de Likert & $\begin{array}{l}\text { Esta teoría indica que las prácticas de gestión } \\
\text { participativa llevan a niveles más elevados de } \\
\text { desempeño organizacional }\end{array}$ & $\begin{array}{l}\text { Lusthaus et al. (2002) } \\
\text { Sandoval (2004) }\end{array}$ \\
\hline $\begin{array}{l}\text { Teoría de } \\
\text { Sistemas }\end{array}$ & $\begin{array}{l}\text { Esta teoría está basada en el hecho de que hay } \\
\text { muchos componentes de una organización o un grupo } \\
\text { que constituyen un sistema. Establece que cada } \\
\text { componente de un sistema es interdependiente, en un } \\
\text { grado algunas veces mayor o menor, de otros } \\
\text { componentes del sistema. }\end{array}$ & $\begin{array}{l}\text { Pinto }(2003) \\
\text { Wallace }(2010)\end{array}$ \\
\hline $\begin{array}{l}\text { Teoría de } \\
\text { contingencia }\end{array}$ & $\begin{array}{l}\text { Esta teoría indica que las empresas u organizaciones } \\
\text { deben de tratar de manejar el nivel de incertidumbre del } \\
\text { entorno por medio de la implementación estrategias, } \\
\text { tanto internas y como externas. Entre las estrategias } \\
\text { internas se encuentra la definición de proceso y } \\
\text { métodos así como estructuras organizacionales que se } \\
\text { adecuen en forma perfecta a las condiciones } \\
\text { ambientales. }\end{array}$ & Pinto (2003) \\
\hline $\begin{array}{l}\text { Teoría de redes } \\
\text { sociales }\end{array}$ & $\begin{array}{l}\text { Esta teoría es una rama de la Microeconomía que } \\
\text { busca como poder predecir el resultado del } \\
\text { comportamiento agregado de un grupo de personas } \\
\text { definido por las relaciones entre sus miembros. }\end{array}$ & $\begin{array}{l}\text { Lozares (1996) } \\
\text { Fuentes (2005) }\end{array}$ \\
\hline $\begin{array}{l}\text { Teoría basada } \\
\text { en los recursos }\end{array}$ & $\begin{array}{l}\text { Esta teoría se basa en la asunción de que una ventaja } \\
\text { competitiva es resultado de una óptima combinación y } \\
\text { asignación de recursos en escenarios imperfectos (con } \\
\text { muchas características adversas). Estos recursos } \\
\text { pueden ser activos, habilidades, y capacidades (de } \\
\text { organización), teniendo todos las características que } \\
\text { todos ellos que llevan a las ventajas competitivas } \\
\text { tienden a ser escasos, valioso, insustituibles y difíciles } \\
\text { de ser imitables. }\end{array}$ & $\begin{array}{l}\text { Penrose (1959) } \\
\text { Wernerfelt (1984) } \\
\text { Barney (1991) }\end{array}$ \\
\hline
\end{tabular}

Fuente: Autoría propia.

\section{En busca de la definición de desempeño organizacional}

Algunos autores como Gopalakrishnan (2000), consideran que el desempeño organizacional tiene amplias definiciones, tales como:

a) La eficiencia, relacionado con el ingreso y el egreso de los recursos de la empresa. 
b) La efectividad, relacionado con el crecimiento del negocio y la satisfacción laboral del empleado.

c) Financiera, relacionado con el retorno de activos, retorno de la inversión, crecimiento de la utilidad.

Otros autores como Lee \& Miller (1996), consideran que el desempeño organizacional depende de los objetivos de la compañía y pueden ser evidenciados por:

a) La rentabilidad de la empresa (utilidad operativa, utilidad operativa sobre activo fijo).

b) El crecimiento evidenciado por incrementos en ventas, ampliación de la cobertura en segmento de mercado, innovación de nuevos productos).

c) La satisfacción de los clientes, y la satisfacción de los empleados (moral y laboral).

Otros enfoques consideran también factores como el crecimiento de ventas, rentabilidad, nuevos productos, segmento de ventas de nuevos productos, segmento de mercado, retorno de capital, y tasa de retorno, Langerak, Hultink \& Robben (2004).

La mayoría de las organizaciones consideran su desempeño en términos del cumplimiento de los mandatos asociados a su misión, sus objetivos o sus metas. Por ejemplo, se considera que una universidad es efectiva en la medida en que proporciona enseñanza, se dedica a la investigación y ofrece un servicio a la comunidad, Lusthaus, Adrien, Anderson, Carden \& Plinio (2002). Se indica que el desempeño organizacional es causado por una combinación de agentes como la efectividad, la eficiencia, la relevancia, lo económico y la viabilidad financiera. Así mismo define la capacidad organizacional, la cual es la habilidad de una organización para utilizar sus recursos en la realización de sus actividades 0 logros de metas y objetivo. Si la unidad de análisis es la organización misma, se pueden evaluar todos los recursos, sistemas y procesos de toda la organización, para apoyarla en su labor. Un examen de los sistemas y prácticas de gestión relacionados con los recursos humanos, financieros y de infraestructura ayuda a comprender el destino o uso que se le están dando a los recursos organizacionales.

El desempeño organizacional de una empresa ha sido medido de diferentes formas propuestas por varios autores, algunos de ellos toman en cuenta razones financieras, indicadores de crecimientos en ventas, tamaño 
de la empresa, cumplimiento de metas y objetivos pero básicamente pudiéramos establecer que es el grado en que las organizaciones 0 empresas cumplen con su trabajo. Algunos de las investigaciones más repetitivas son expuestas en la siguiente tabla, Gibb (2006), ver Tabla 4.

Tabla 4. Medidas de desempeño organizacional

\begin{tabular}{|c|c|}
\hline Autor & Medidas de Desempeño \\
\hline Anderson y Reeb (2003) & $\begin{array}{l}\text { 1. Tobin's q. } \\
\text { 2. Retorno en activos. } \\
\text { 3. Retorno en capital. }\end{array}$ \\
\hline $\begin{array}{l}\text { Beehr, Drexler y Faulkner } \\
\text { (1997) }\end{array}$ & $\begin{array}{l}\text { 1. Conflicto: conflictos trabajo-familia / conflictos interpersonales. } \\
\text { 2. Expectativas y ventajas: expectativas familiares / ventajas } \\
\text { personales. } \\
\text { 3. Resultados o indicadores individuales: satisfacción en el trabajo / } \\
\text { satisfacción de la carrera / tensión o estrés psicológico. } \\
\text { 4. Resultados o indicadores Organizacionales: compromisos } \\
\text { organizacionales /volumen de ventas } \\
\text { 5. Resultados o indicadores familiares: harmonía familiar. }\end{array}$ \\
\hline $\begin{array}{l}\text { McConaughy, Matthews y } \\
\text { Fialko (2001) }\end{array}$ & $\begin{array}{l}\text { 1. Eficiencia. } \\
\text { 2. Estructura de Capital. } \\
\text { 3. Valor. }\end{array}$ \\
\hline $\begin{array}{l}\text { Gallo, Tapies y Cappuyns } \\
(2000)\end{array}$ & Crecimiento, deuda y otras medidas financieras. \\
\hline Villalonga y Amit (2004) & Tobin's q. \\
\hline $\begin{array}{l}\text { Tanewski, Prajogo y Sohal } \\
(2003)\end{array}$ & $\begin{array}{l}\text { 1. Innovación de productos. } \\
\text { 2. Innovación de procesos. } \\
\text { 3. Estructura. } \\
\text { 4. Estrategia del líder. }\end{array}$ \\
\hline
\end{tabular}

Fuente: Tomado de Gibb (2006).

Para el estudio de la relación motivo de este trabajo proponemos el siguiente concepto de desempeño organizacional: de una empresa que usaremos en la presente investigación es que está definido por el cumplimiento de metas económico-financieras.

\section{El grado de control familiar}

El efecto de la familia o el grado de control familiar son aquellos atributos o características que la familia propietaria de una empresa lleva a 
ella y que en determinado momento pueden afectar su desempeño organizacional. Este desempeño organizacional podemos definirlo ampliamente en términos de eficiencia, en términos de utilización de recursos de forma eficiente así como el cumplimiento de metas organizacionales, Steers (1982). Las familias propietarias afectan el desempeño organizacional de la empresa por medio de varios aspectos como son las metas propias de la familia, sus relaciones (con proveedores, accionistas, etc.) y los recursos 0 activos familiares que posee.

Para desarrollar una teoría acerca de cómo las metas u objetivos familiares, las relaciones y los recursos afectan el desempeño organizacional de una empresa, lo más conveniente es partir de la teoría de agencia y de la teoría basada en los recursos, Chrisman, Chua \& Litz (2004); estas dos teorías sugieren ciertos factores familiares pueden acarrear beneficios de agencia e importantes activos, mientras otros factores familiares imponen costos que se convierten en pasivos y son barreras para el óptimo desempeño de la empresa, Gibb (2006). Este mismo autor indica que los factores familiares que contribuyen al desempeño de la empresa, como los beneficios derivados de la teoría de agencia (bajos costos) y los relacionados a los activos de la familia; por otro lado también hay factores familiares que contribuyen al bajo de desempeño de la empresa.

\section{Definición de control familiar}

Con base en lo anterior expuesto, para la presente investigación se usará como definición de grado de control familiar el impacto que tiene la influencia de la familia propietaria de una empresa en los procesos estratégicos y en el desempeño organizacional de la misma.

\section{Algunas teorías de Control Familiar y Desempeño Organizacional.}

La mayoría de las investigaciones acerca de la empresa familiar se han basado en el marco teórico que se cimenta en la exposición de la teoría de la agencia, la teoría de capital social y la teoría de Familiness. Un resumen de las principales trabajos empíricos se presenta continuación, ver Tabla 5. 
Tabla 5. Resumen de teorías de control familiar

\begin{tabular}{|c|c|c|}
\hline Teoría & Descripción & Autores \\
\hline $\begin{array}{l}\text { Teoría de la } \\
\text { agencia }\end{array}$ & $\begin{array}{l}\text { Es una teoría económica que trata las relaciones } \\
\text { agente/principal, es decir, trata las relaciones que } \\
\text { surgen cuando en una empresa la autoridad o } \\
\text { responsabilidad para la toma de decisiones es } \\
\text { delegada, como lo comenta. }\end{array}$ & $\begin{array}{l}\text { Daily y Dollinger (1992) } \\
\text { Habbershon \& Williams (1999) } \\
\text { Salinas (1999) } \\
\text { Reyes y Sacristán (2003) } \\
\text { Miller \& Le Breton-Miller (2006) } \\
\text { Tsai, Hung, Kuo, \& Kuo (2006) } \\
\text { Blanco Mazagatos et al. (2007) } \\
\text { Sánchez Gardey et al. (2009) }\end{array}$ \\
\hline $\begin{array}{l}\text { Teoría de } \\
\text { capital social }\end{array}$ & $\begin{array}{l}\text { Esta teoría provee un marco de referencia para } \\
\text { identificar las capacidades y recursos conductuales } \\
\text { de las empresas familiares, así como sus } \\
\text { antecedentes históricos de capital social. } \\
\text { Específicamente el historial familiar sirve como una } \\
\text { condicionante histórica y única que altera la manera } \\
\text { como la familia influencia a los procesos estratégicos } \\
\text { y al desempeño de la empresa }\end{array}$ & $\begin{array}{l}\text { Lyman(1991) } \\
\text { Adler \& Kwon (2002) } \\
\text { Gibb (2006) } \\
\text { Pearson (2008) }\end{array}$ \\
\hline $\begin{array}{l}\text { Teoría de } \\
\text { Familiness }\end{array}$ & $\begin{array}{l}\text { Esta teoría se deriva de la teoría de capital social se } \\
\text { define como la intersección de la familia y la } \\
\text { empresa, específicamente identifica los recursos } \\
\text { sociales y conductuales que son originados o } \\
\text { resultantes cuando la familia propietaria de una } \\
\text { empresa se compromete y se enfoca al } 100 \% \text { en ella. }\end{array}$ & $\begin{array}{l}\text { Olson et al. (2003) } \\
\text { Pearson (2008) }\end{array}$ \\
\hline
\end{tabular}

Fuente: autoría propia

La teoría de la agencia ha sido la base muchas de las investigaciones acerca de la empresa familiar, resaltando que la mayor parte de los análisis han sido planteados en grandes empresas como lo indica según Sánchez Gardey et al. (2009). La teoría de la agencia es una teoría económica que trata las relaciones agente - principal, es decir, trata las relaciones que surgen cuando en una empresa la autoridad o responsabilidad para la toma de decisiones es delegada, como lo comenta Salinas (1999). Esta teoría se enfoca en dos figuras muy importantes dentro de la empresa: los dueños y los administradores (gerentes). Un ejemplo de esto es cuando los accionistas (dueños) de una empresa delega su autoridad para la toma de decisiones al personal administrador de la misma, quienes finalmente son sólo empleados y no poseen ningún porcentaje de propiedad de la empresa en cuestión. Se indica que los administradores de las grandes corporaciones, al no ser 
dueños de un porcentaje de la empresa, presentan mayor disposición para beneficiarse con algunos aspectos o recursos de la organización, los cuales representan grandes gastos y no le aportan ningún beneficio organizacional 0 de valor agregado a la misma, Clark (1986).

La teoría de capital social provee un marco de referencia para identificar las capacidades y recursos conductuales de las empresas familiares, así como sus antecedentes históricos de capital social. Específicamente el historial familiar sirve como una condicionante histórica y única que altera la manera como la familia influencia a los procesos estratégicos y al desempeño de la empresa. La teoría de capital social ha sido aplicada para analizar la gran variedad de relaciones sociales colectivas en, además de empresas familiares, equipos de trabajo, subsidiarias o ramas de negocios, etc. y se conceptualiza en tres dimensiones: estructural, cognitivo y relacional, Pearson (2008).

Respecto de la Teoría de Familiness, esta teoría se deriva de la teoría de capital social se define como la intersección de la familia y la empresa, específicamente identifica los recursos sociales y conductuales que son originados o resultantes cuando la familia propietaria de una empresa se compromete y se enfoca al $100 \%$ en ella, Olsonet al. (2003) y Pearson (2008).

Sacristán, Forcadell \& Montero (2003) definen el Familiness como "aquel conjunto de recursos que son distintivos de una empresa como resultado de la implicación en ésta de una familia". Esta formulación apoya la utilización de los distintos conceptos de la teoría de los recursos y capacidades para el análisis de las empresas familiares. De este modo, podemos identificar una empresa familiar como una organización que posee un conjunto específico de recursos o capitales, distintos de otras organizaciones cuyo carácter no es familiar. Dichos recursos procederán de la vinculación entre las características de la empresa y la familia.

Como se mencionaba antes, hay tres tipos de capital (activos) que han sido asociados al desempeño de la empresa familiar, $\operatorname{Gibb}(2006)$ :

- Capital social.

- Capital humano.

- Capital físico y financiero.

El capital social es un fenómeno y una forma simple de definirlo es que es el beneficio que resultado de la realización de relaciones sociales y que 
puede ser trasladado para facilitar su aplicación para un fin determinado, Adler \& Kwon (2002) y Gibb (2006). El capital social es un importante activo que le permite a la empresa tener acceso a otros activos por medio de alianzas, contratos, convenios y otras formas de acuerdos.

El capital social es un importante activo que permite a la compañía ganar acceso a otras formas de capital (o riqueza) como el capital humano, financiero o intelectual, los cuales son lo que realmente la empresa necesita para sobrevivir y cumplir sus metas de desempeño organizacional. Algunas otras ventajas generadas por el capital social proveniente del control familiar sobre es una empresa es la posible facilidad de conseguir más clientes duraderos basados en el bienestar y confianza del buen nombre de la familia propietaria y en el compromiso en servicio al cliente, Lyman (1991). Otros ejemplos de capital social el bienestar del empleo duradero. Por ejemplo el reporte de Meek et al. (1988) en su estudio de huelgas ocurridas en Jamestown, New York, reportó que las compañía con propietarios en locales, la mayoría con conexiones familiares, tuvieron significativamente menos huelgas y las que llegaron a suceder tuvieron un tiempo de duración significativamente menor que las compañías foráneas (con propietarios no locales), Meek, Woodworth \& Dyer (1988).

Cabe mencionar que así como hay ventajas también hay desventajas derivadas del capital social. La existencia de enlaces familiares fuertes también tiene desventajas como cuando esos lazos familiares demasiado fuertes hacen que se vea a todos los agentes externos como una amenaza 0 como competidores, impidiendo de esta forma a la empresa poder llevar a cabo alianzas, sinergias, convenios, etc., las cuales pudieran haber contribuido al cumplimiento del desempeño organizacional.

La teoría de capital social provee un marco de referencia para identificar las capacidades y recursos conductuales de las empresas familiares, así como sus antecedentes históricos de capital social. Específicamente el historial familiar sirve como una condicionante histórica y única que altera la manera como la familia influencia a los procesos estratégicos y al desempeño de la empresa. La teoría de capital social ha sido aplicada para analizar la gran variedad de relaciones sociales colectivas en, además de a empresas familiares, equipos de trabajo, subsidiarias 0 ramas de negocios, etc. y se conceptualiza en tres dimensiones: estructural, cognitivo y relacional, Pearson (2008). 
Respecto del capital humano, podemos establecer que es uno de los recursos que le puede dar a una compañía ventajas competitivas. Es tangible por medio de las capacidades, las habilidades, las actitudes y la ética de trabajo de los empleados de la compañía. Hay varias situaciones por qué los investigadores indican que las empresas familiares tienen un gran valor de capital humano. Primera, porque "el nombre de la familia está en el edificio"; lo anterior generará que los miembros de la familia que trabajan en la empresa se sentirán naturalmente más comprometidos con los objetivos y metas de las misma. Segunda, debido a que los miembros de la familia frecuentemente han socializado desde la niñez y están muy familiarizados con la empresa y su visión y misión, es decir, ya conocen la naturaleza del negocio. Así mismo ha generado los lazos para poder capitalizar este capital humano en capital social que a su vez pueda ser generador de riqueza para la empresa como en los ejemplos ya comentados. Tercero, la lealtad desarrollada podrá generar fuerza laboral que esté dispuesta a trabajar largas jornadas, en ocasiones sin un incentivo monetario extra.

Por otro lado, en las empresas familiares se podría estar limitado para poder satisfacer con miembros de la familia las necesidades de personal calificado para puestos claves. Esto puede llegar a generar problemas sí se conjunta con fenómenos como el nepotismo a la hora de cubrir una vacante en un puesto de alta especialización. Empleados familiares incompetentes podrían estar siendo asignados a esos puestos clave lo que podría poner en riesgo el poder al cumplir con el desempeño organizacional esperado.

Respecto del capital financiero y físico, este se basa en que la familia propietaria de una empresa podría inyectar recursos financieros para tratar de poder contribuir al desarrollo de la empresa (con el objetivo de tratar de lograr las metas de desempeño establecidas), sin por hacer esto estar asegurándolo. Pero no siempre ocurre lo anterior, es un estudio de 673 empresas familiares (Haynes, Walker, Rowe, \& Hong, 1999)se le pregunto a los dueños que describieran si los fondos o activos de la familia habían sido usados para apuntalar la empresa en determinado momento o si por el contrario el negocio era usado para satisfacer las necesidad financieras de la familia. Este estudio concluyó que es mucho más frecuente que la familia extraiga recursos de la empresa para satisfacer sus necesidades que los fondos de la familia sirvan de soporte a la empresa. 


\section{Estudios empíricos de control familiar y desempeño de la empresa}

Se han llevado a cabo estudios de la relación que existe en el desempeño organizacional y el grado de control familiar. En la búsqueda de la información para este estudio se han encontrado algunos trabajos de gran relevancia que veremos a continuación.

Los principales estudios empíricos que se han realizado sobre sí el control familiar y el desempeño organizacional están correlacionados están indicados en las Figuras 1 y Figura 2. A continuación se muestran los principales resultados detectados en dichos estudios empíricos.

Figura 1. Estudios empíricos sobre control familiar

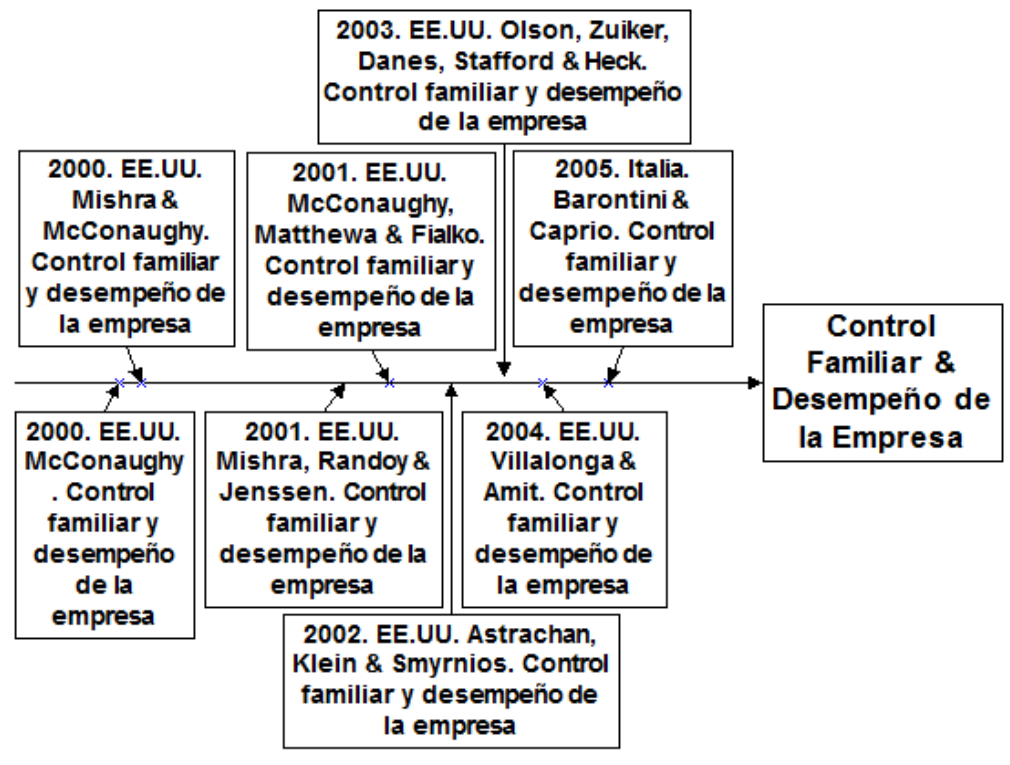

Fuente: Autoría propia. 
Figura 2. Estudios empíricos sobre control familiar

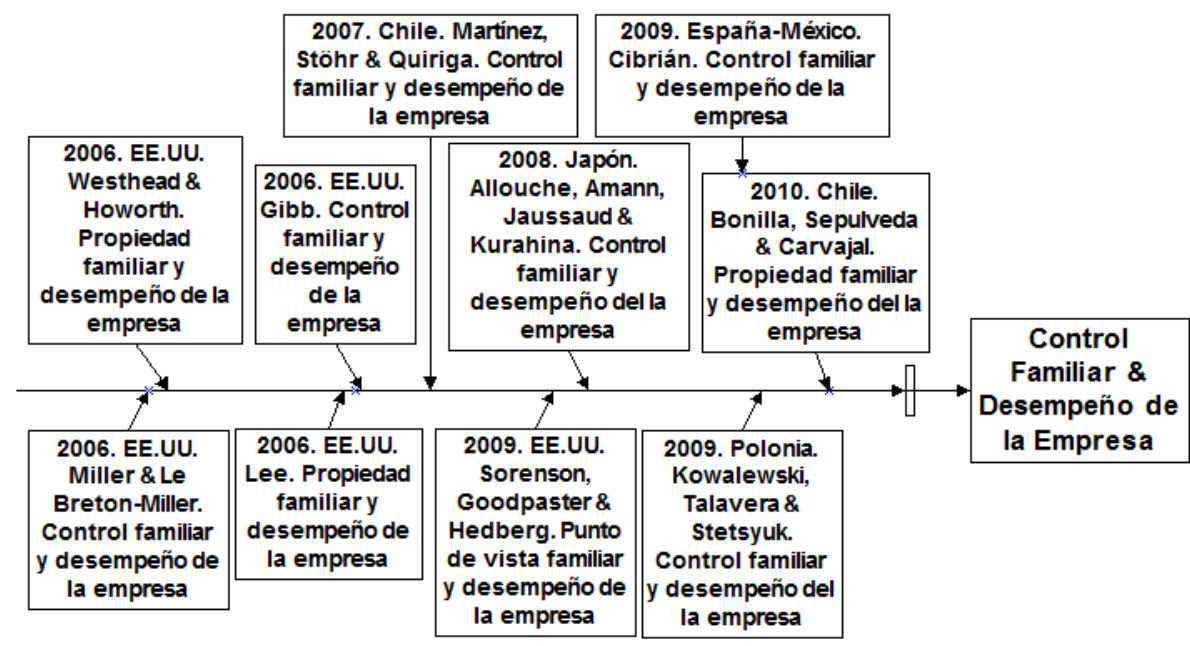

Fuente: Autoría propia.

Respecto de los estudios empíricos anteriores, Cibrián (2009) concluyó que la teoría del capital social es un marco teórico que permite explicar cómo las familias intervienen en sus empresas; esta misma autora indica que la influencia de la familia puede ser positiva o negativa para el desempeño de la empresa dependiendo por ejemplo de que la familia tenga relaciones funcionales, que su red de vínculos sea amplia y profunda, que fomenten un nivel de confianza adecuado entre los integrantes de la empresa y diseñen normas que motiven el trabajo en equipo.

Por su parte Sorenson, Goodpaster, Hedberg, \&Yu (2009) indican que hay una significante relación positiva entre el capital social y el desempeño de la empresa además señalan que la ventaja estratégica para las empresas familiares reside en construir fuertes redes de relaciones. Westhead \& Howort (2006) encontraron en sus análisis que las empresas que tiene un alto grado de propiedad o control familiar no están significativamente relacionadas con niveles de desempeño organizacional débil.

Por su parte Miller \& Le Breton-Miller (2006) encontraron que las empresas de propiedad familiar tienen un mejor desempeño debido a costos 
de agencias más bajos. Kowalewski, Talavera \& Stetsyuk (2010) en Polonia encontraron que la propiedad y la administración familiar de una empresa están relacionados de manera positiva y significativa con el desempeño financiero de la empresa, esto es congruente con lo encontrado por Villalonga \& Amit (2004) acerca de la influencia positiva del control familiar en el desempeño de una empresa. Así mismo Mishra, Randoy \& Jenssen (2001) encontraron que hay relación positiva entre el control familiar y el valor de la empresa, al igual que McConaughy (2000). Estos mismos investigadores encontraron que el control familiar es más apreciado entre empresas nuevas.

Martínez, Stöhr \& Quiroga en (2007) y más tarde Bonilla, Sepúlveda, \& Carvajal (2010) encontraron en Chile que las empresas familiares tiene un desempeño mejor que las empresas que no lo son (esto debido al control familiar que tiene la empresa), siendo lo anterior congruente con el caso de Japón mostrado por Allouche, Amann, \&Kurash (2008) y Kurashina (2003). En Italia en 2005, Barontini y Caprio encontraron que el control familiar está claramente asociado con un mejor control del flujo de dinero en las empresas.

Así mismo, Lee (2006) encontró que las empresas familiares pueden tener un mejor desempeño si los miembros fundadores participan en la administración de las mismas, sumado a que no hay evidencia de que a pesar de su crecimiento más rápido sean menos estables que las que no son familiares (no hay grado de control familiar en ellas).

Por su parte Gibb (2006) indica que dependiendo del tipo de empresa familiar de que se trate y su grado de control relacionado será el nivel de desempeño organizacional que pueda alcanzar una empresa. Sumado a lo anterior, Villalonga (2004) que la empresa familiar tiene ventajas competitivas y mejores beneficios que la empresa que no lo es. McConaughy et. al (2001) demostraron que las empresas familiares controladas por la generación fundadora tiene un desempeño mayor que las empresas familiares controladas por generaciones subsecuentes a la fundadora y mayor también que las empresas no familiares.

\section{Proposiciones / hipótesis}

A continuación se expone la hipótesis del presente artículo de investigación. 
Proposición / Hipótesis específicas de investigación: El grado de control familiar que ejerce la familia propietaria sobre la empresa MIPyME familiar está relacionado de manera directa y positiva con el desempeño organizacional de dicha empresa.

\section{Conclusión}

Las empresas familiares son la forma más común de organización de negocio. Sus características únicas incluyen los altos niveles de confianza y de compromiso, que pueden dar lugar a mayor eficacia y mayores beneficios que las empresas administradas por los propietarios diversos. El resultado del análisis conceptual nos permite suponer que se pueda comprobar que el grado de control familiar que ejercer la familia propietaria de una empresa sobre la misma tenga características similares a las presentas en los estudios empíricos revisados. Por lo tanto proponemos que se pruebe la proposición con la que se concluye este estudio conceptual.

\section{Bibliografía}

Allouche, J., Amann, B., \& Kurash, T. (2008). The Impact of Family Control on the Performance and Financial Characteristics of Family Versus Nonfamily Businesses in Japan: A Matched-Pair Investigation. Family Business Review, 21(4), 315-329.

Astrachan, J., \& Carey, M. (2003). Family businesses' contribution to the U.S. economy: A closer look. Family Business Review, 16(3), 211-219.

Barney, J. (1991). Firm Resource and Sustained Competitive Advantage. Journal of Management, 17(1), 99-120.

Belausteguigoitia, I. (2005). La Influencia de la Familia en las Organizaciones Familiares Mexicanas. México D.F.: Centro de Desarrollo de la Empresa Familiar Instituto Tecnológico Autónomo de México (ITAM).

Belausteguigoitia, I. (2005). Luz y sombra en las Empresas Familiares. México D.F.: Centro de Desarrollo de la Empresa Familiar Instituto Tecnológico Autónomo de México (ITAM).

Bonilla, C., Sepulveda, J., \& Carvajal, M. (2010). Family Ownership and Firm Performance in Chile: A Note on Martinez et al.'s Evidence. Family Business Review, 23(2), 148-155.

Braun, M., \& Sharma, A. (2007). Should the CEO also be the Chair of the Board? Family Business Review, 20(2), 111-126.

Chrisman, J. J., Chua, J. H., \& Litz, R. A. (2004). Comparing the agency cost of family and non-family firms, Entrepreneurship Theory and Practice, 28(4), 335-354. 
Cibrián, T. (1 de Enero de 2009). Influencia de la familia en el desempeño de las PYMES familiares mexicanas. Barcelona, Cataluña, España.

CIPI de la SE. (2003). Primer Reporte de Resultados 2002. México D.F.: Secretaría de Economía del Gobierno Federal.

Clark, F. (1986). Investments: Analysis and management. Atlanta: McGraw-Hill.

Craig, J., \& Dibrell, C. (2009). The Natural Environment, Innovation, and Firm Performance: A Comparative Study. Family Business Review, 19(4), 275-288.

Fabelo, J. R. (2001). Los valores y la familia. En J. R. Fabelo, Los valores y los desafios actuales. México: BUAP.

Anderson, R.C. \& Reeb, D.M. (2003). Founding-family ownership and firm performance: evidence from the S\&P 500.2003. The Journal of Finance, 58(3), 1301-1328.

Fuentes, F. (2005). La ciencia de la administración de empresas: un análisis de sus componentes y de la contribución de la revista economía y administración. Economía y Administración, 1(64), 1-28.

Gallo, M. A. (1995). Empresa familiar, textos y casos. Barcelona: Praxis.

Gersick, K. E. (1997). Generation to Generation. Boston: Harvard Business School Press.

Gibb, W. (2006). Examining the Family Effect on Firm Performance. Family Business Review, 19(4), 253-263.

Gonzalez, J. M. (2007). La familia como un sistema. Revista Paceña de Medicina Familiar, 4(6), 111-114.

Gopalakrishnan, S. (2000). Unraveling the Links Between Dimensions of innovation and Organizational Performance. The Journal of High Technology Management Research, 11(1), 137-153.

Grabinsky, S. (1991). La empresa familiar, guía para crecer y sobrevivir. México D.F.: México: Del Verbo Emprender.

Haynes, G. W., Walker, R., Rowe, B. R., \& Hong, G. (1999). The intermingling of business and family finances in family-owned businesses. Family Business Review, 12(3), 225-239.

INEGI. (2010). Encuesta Nacional de Ocupación y Empleo (Segundo Trimestre). México, D.F.: INEGI.

INEGI. (2010). Informe de Censo Economico 2009. México, D.F.: INEGI.

Kajihara, K. (2004). Las Empresas Familiares: La Realidad Empresarial Mexicana. México D.F.: Centro de Dirección Estratégica del Instituto Tecnológico Autónomo de México (ITAM).

Kowalewski, O., Talavera, O., \& Stetsyuk, I. (2010). Influence of Family Involvement in Management and Ownership on Firm Performance: Evidence From Poland. Family Business Review, 23(1), 45-59.

Kurashina, T. (2003). Management studies on family business. Family Business Review, 150162.

Langerak, F., Hultink, E., \& Robben, H. (2004). The Impact of Market Orientation, Product Advantage, and Launch Proficiency on New Product Performance and Organizational Performance. Journal of product Innovation Management, 19(2), 79-94. 
Lee, J. \& Miller, D. (1996). Strategy, Environment and Performance in Two Technological Contexts: Contingency Theory in Korea, Organizational Studies, 17(5), 729-750

Lozares, C. (1996). La teoria de redes sociales. Papers 48, 1(1), 103-126.

Lusthaus, C., Adrien, M.-H., Anderson, G., Carden, F., \& Plinio, G. (2002). Evaluación organizacional. Ottawa: Centro Internacional de Investigaciones para el Desarrollo.

Lyman, A. R. (1991). Customer service: Does family ownership make a difference? Family Busines Review, 4(3), 303-324.

Martínez, J., Stöhr, B., \& Quiroga, B. (2007). Family Ownership and Firm Performance: Evidence from Public Companies in Chile. Family Business Review, 20(2), 83-94.

McConaughy, D. (2000). Family CEOs vs. Nonfamily CEOs in the Family-Controlled Firm: An Examination of the Level and Sensitivity of Pay to Performance. Family Business Review, 13(2), 121-132.

McConaughy, D., Mendoza, D., \& Mishra, C. (1996). Loyola University Chicago Family Firm Stock Index. Family Business Review, 9(2), 125-137.

Meek, C., Woodworth, W., \& Dyer, W. G. (1988). Managing by the numbers: Absentee owners and the decline of American industry. Reading, MA: Addison-Wesley.

Miller, D., \& Le Breton-Miller, I. (2006). Family Governance and Firm Performance: Agency, Stewardship, and Capabilities. Family Business Review, 19(1), 73-87.

Morales, M. A. (2009). Modelos de formación para la capacitación de las familias en la sociedad de la información. (Doctoral dissertation). Granada, España: Editorial de la Universidad de Granada.

Müller-Lietzkow, J. (1991). Free Resources and the resource-based view. Media economics, 493-498.

Olson, P., Zuiker, V., Danes, S., \& Stafford, K. (2003). Impact of family and business on family business sustainability. Journal of Business Venturing, 18(5), 639-666.

Pearson A., C. J. (2008). Toward a theory of familiness: A social capital perspective. Entrepreneurship Theory and Practice, 32(6), 949-969.

Penrose, E. (1959). The theory of the growth of the firm. Oxford: Oxford University Press.

Poza, E. (2007). Family Business. Whasington, D.C.: Thomson South-Western.

Prencipe, A., Markarian, G., \& Pozza, L. (2008). Earnings Management in Family Firms: Evidence from R\&D Cost Capitalization in Italy. Family Business Review, 21(1), 7188.

Rendón, M. V. (2010). Empresa familiar y configuraciones organizacionales. México, D.F.: Universidad Autónoma Metropolitana-Iztapalapa.

Sacristán, M. A., Forcadell, F. J., \& Montero, A. (2003). ¿Qué es Familiness? Hacia una teoría de la empresa familiar. Madrid: Universidad Rey Juan Carlos.

Salinas, L. d. (1999). Análisis comparativo de las diferencias en la situación financiera de la empresa familiar y de la empresa no familiar de tamaño medio que cotizan en bolsa dentro del mercado para la mediana empresa mexicana: un caso de estudio en México. Instituto Tecnológico y de Estudios Superiores de Monterrey, Monterrey, México. , 1-150.

Secretaría de Economía (2009).Acuerdo por el que se establece la estratificación de las micro, pequeñas y medianas empresas, Diario Oficial de la Federación: 30/06/2009. 
Sorenson, R. L., Goodpaster, K. E., Hedberg, P. R., \& Yu, A. (2009). The Family Point of View, Family Social Capital, and Firm Performance: An Exploratory Test. Family Business Review, 22(3), 239-253.

Steers, R. (1982). When is an organization effective? En R. Steers, Designing effective organizations (págs. 196-206). Lexington, MA: E. F. McDonough.

Tsai, W.-H., Hung, J.-H., Kuo, Y.-C., \& Kuo, L. (2006). CEO Tenure in Taiwanese Family and Nonfamily Firms: An Agency Theory Perspective. Family Business Review, 19(1), 1128.

Villalonga, B., \& Amit, R. (2004). How do family ownership, control and management affect firm value? Journal of Financial Economics, 80(2), 385-417.

Wallace, J. S. (2010). Family-owned businesses: Determinants of business success and profitability. All Graduate Theses and Dissertations , 123-129. Utah, USA: Utah State University.

Wernerfelt, B. (1984). A resource based view of the firm. Strategic Management Journal, 5(1), 171-180.

Westhead, P., \& Howorth, C. (2006). Ownership and Management Issues Associated With Family Firm Performance and Company Objectives. Family Business Review, 19(4), 301-316. 